\title{
Percolation transition in a dynamically clustered network
}

\author{
A. Zen, ${ }^{1,2, *}$ A. Kabakçıŏlu, ${ }^{3}$ and A. L. Stella ${ }^{1,2}$ \\ ${ }^{1}$ Dipartimento di Fisica, Università di Padova, I-35131 Padova, Italy \\ ${ }^{2}$ Sezione INFN, Università di Padova, I-35131 Padova, Italy \\ ${ }^{3}$ Department of Physics, Faculty of Arts and Sciences, Koç University, Rumelifeneri Yolu 34450, Sariyer Istanbul, Turkey
}

(Received 21 March 2007; published 20 August 2007)

\begin{abstract}
We consider a percolationlike phenomenon on a generalization of the Barabási-Albert model, where a modification of the growth dynamics directly allows formation of disconnected clusters. The transition is located with high precision by an original numerical technique based on the comparison of the largest and second largest clusters. A careful investigation focusing on finite size scaling allows us to highlight properties which would hardly be accessible by an analytical solution of cluster growth equations in the stationary limit. Our analysis shows that some critical features of the percolation transition are different from those observed in the case of dilution in fully grown networks. At variance with other models of percolation on growing networks we also find evidence that the order parameter approaches zero as a power of the field $p-p_{c}$ driving the transition, rather than as a stretched exponential. This behavior does not agree with the Berezinskii-KosterlitzThouless scenario found in other similar models. For describing the phase in which a giant cluster develops, a key role is played by the crossover number of nodes $N_{x} \sim\left(p-p_{c}\right)^{-\zeta}$ with $\zeta \simeq 4$. This power law behavior and that of other quantities are conjectured on the basis of scaling arguments and numerical evidence.
\end{abstract}

DOI: 10.1103/PhysRevE.76.026110

PACS number(s): 89.75.Hc, 64.60.Fr, 89.75.Fb

\section{INTRODUCTION}

Following the enormous interest in the network description of many real-life complex systems [1,2], the percolation transition on these models has become of interest as well. One motivation for studying the percolation phenomenon on networks is its connection to epidemic spreading $[3,4]$. The existence of disconnected clusters in a variety of networks, such as the protein interaction [5] and metabolic [6,7] networks in cells or the network of daily telephone calls in the U.S. [8] demands a deeper understanding into the nature of such disconnectivity. As a further example, the connectivity of the "incompatibility graph" of a polymer can be tuned by changing the temperature, where a dissolvation phenonmenon akin to percolation can be shown to accompany the $\theta$ transition [9]. Absence of interactions that undermines a connected topology may also stem from the fact that a fraction of the network is yet to be discovered [10].

Real-life networks may be viewed as imperfect realizations of idealized theoretical models. We are familiar, e.g., from earlier studies of magnetic materials, with the fact that introducing defects (impurities) into such a model may dramatically alter its character [11]. In the case of large-scale networks, e.g., the Internet, power grids, social contacts, a parallel approach is used to study communication failures due to damaged nodes $[12,13]$. The connectivity of such networks as a function of node/edge removal probability is well-studied analytically [14-16].

In this article we focus on a different percolation mechanism that is realized in growing networks. Instead of clusterization by dilution of a fully grown network, we define an imperfect growth process that generates disconnected com-

*Present address: International School for Advanced Studies (SISSA), Via Beirut 2-4, 34014 Trieste, Italy. ponents on the fly. The related percolation transition is dynamic in essence and shows features distinctly unlike its dilution-origined counterpart. Our analysis is not based on the analytical solution of the equations governing cluster formation in the stationary, infinite time limit. Unlike for previously studied models [17], these equations are not easily solvable in our case. As a consequence, we have to resort to a purely numerical analysis based on finite size scaling. The obvious limitations of a numerical approach do not prevent us from reaching solid conclusions about the nature of the transition and of the various asymptotic laws. On the contrary, dealing with finite size systems opens the possibility of studying regimes and scaling behaviors seldomly exploited in the literature on networks $[18,19]$, although they are of particular importance in practice. In this respect, our contribution should also have some general methodological value.

\section{MODEL}

We consider here a natural generalization of the BarabásiAlbert (BA) model [20] that introduces an imperfection into the growth process, thereby allowing formation of clusters. As in the BA model, the network grows by the addition of a single node at each time step. The subsequent behavior of the growth dynamics is determined probabilistically through a parameter $p(0 \leqslant p \leqslant 1)$. With probability $(1-p)$, the newly introduced node behaves identical to the unperturbed BA model, i.e., stretches out $m$ connections to existing nodes. A node is selected with a probability $P(k)$ related to the number of edges $k$ it acquired after birth, which we will refer to as its "degree" (dropping the "in" prefix for brevity). As is standard for BA-like models, we set $P(k) \propto k+A$. Otherwise, with probability $p$, the node remains initially isolated. However, since $A>0$, it may still acquire connections from the nodes introduced at later stages. In the limits $p=0$ and $p=1$ the model reduces to a singly connected network (original BA 


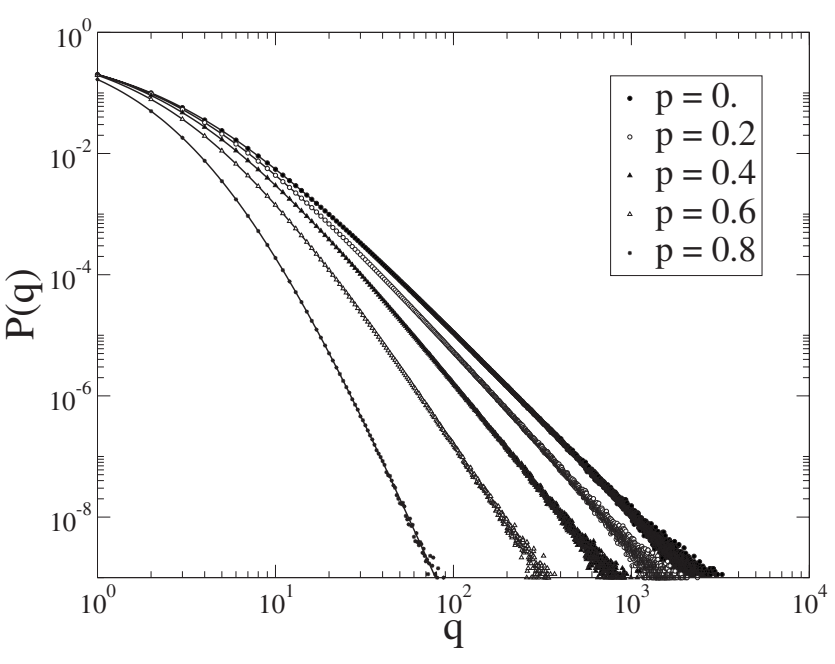

FIG. 1. The degree distributions for $A=m=2, N=2^{23}$, and various values of $p$. The continuous lines correspond to the analytical solution of the master equation given in Eq. (2).

model) and a fully disconnected network, respectively.

The total number of nodes at time step $t$ is $N=t+m_{0}$, where the starting configuration is composed of $m_{0}$ unconnected nodes. When $N$ is sufficiently large such that $N$ $\gg 1 /(1-p)$ and $N \gg m_{0}$, the number of edges is approximately $(1-p) m N$ and the average degree of a node is $\bar{k}=(1$ $-p) m$. We take $A=m=m_{0}=2$ throughout this paper.

This generalization of the BA model is a natural setup to investigate the formation of disconnected clusters as part of the growth dynamics. It was shown earlier that such dynamic cluster generation models [14,17] exhibit features distinct from dilution-based cluster generation models [15] in the vicinity of the associated percolationlike transition. We show here that, the cluster statistics of the introduced model may be investigated through an original numerical technique. By simulating networks up to size $N=2^{23}$, we furthermore provide an accurate picture of the finite size effects (which are not easily accessible by the steady-state analysis of the related master equation), as well as the thermodynamic ( $N$ $\rightarrow \infty$ ) limit in these models. We find that the percolative nature of the associated transition is distinct from that of Ref. [17] where the introduction of nodes and edges are decoupled from each other.

The characteristic aspects of the network that we will focus on are the degree and the cluster size distributions. The degree distribution $P_{d}(q, N, p)$ will serve as an indicator for how the scale-free character of the system transforms in the process, whereas the cluster size distribution $P_{\mathrm{cl}}(s, N, p)$, which starts from a delta function at $s=N$ when $p=0$ and eventually develops into a power law at large $p$ values, will allow us to monitor the percolationlike phase transition mentioned above.

\section{DEGREE DISTRIBUTION}

Figure 1 shows the behavior of the average degree distribution with changing $p$. Our first observation is that while increasing $p$ from zero up, the power $\gamma$ describing the degree distribution of the vertices in the model deviates appreciably from the BA value $\gamma=3$. This dependence can be obtained analytically by generalizing the master equation for the evolution of the degrees in our model. The new master equation reads

$$
p(q, \tau, t+1)=p p(q, \tau, t)+(1-p) \sum_{l=0}^{m} \mathcal{P}_{\tau, t}^{(m l)} p(q-l, \tau, t),
$$

where $p(q, \tau, t)$ is the probability that the node $\tau$ has $q$ connections at time $t$. The last term accounts for the possible increase of the degree of the node $\tau$ in the event (with a likelihood of $1-p$ ) that the node introduced at time $t$ extends out $m$ connections. The sum is over possible increments $l$ in the degree, weighted by $\mathcal{P}_{\tau, t}^{(m l)}$, the probability that $l$ out of the $m$ connections extend to the node $\tau$. This master equation is identical to that of the model introduced by Dorogovtsev et al. [17] and has the steady-state solution

$$
P_{d}(q)=(1+\widetilde{a}) \frac{\Gamma(1+A+\widetilde{a})}{\Gamma(A)} \frac{\Gamma(q+A)}{\Gamma(q+2+A+\widetilde{a})},
$$

where $\Gamma(x)$ is Euler's gamma function. We refer the reader to Ref. [17] for a derivation. Equation (2) asymptotically approaches the power law

$$
P_{d}(q) \propto(q+A)^{-\gamma(p)}
$$

with an exponent $\gamma(p)=2+\widetilde{a}=2+\frac{A / m}{1-p}$.

The agreement between the simulation values and Eq. (2), as shown in Fig. 1, serves as a check on the accuracy of our simulation results, which we later will rely on during our investigation of the percolative aspects. Also note that the degree distribution is calculated over all the vertices and not over, say, the largest connected cluster, which at first glance may appear as an ideal BA network (but it is not, since its growth dynamics involves merging clusters). Nevertheless, for small $p$, an overwhelming fraction of the nodes belong to the largest cluster and the distinction is hardly visible. As $p$ gets closer to unity, the dominance of the largest cluster disappears with a phase transition, akin to percolation. In the next section, we discuss the details of this regime.

\section{THE GIANT CLUSTER}

Unlike the degree distribution, the cluster size distribution of the original BA network is trivial since there is only one cluster which is of size $N=t$ at any time $t$. The imperfect growth process we introduce here allows formation of multiple disconnected networks when $p>0$, although a giant cluster (GC) which encompasses a finite fraction of all the nodes still dominates the network. The chances for such satellite clusters to survive (i.e., not to merge with the giant cluster) is inversely proportional to their sizes. Therefore, even when $p=0.9$, the distribution of these satellite clusters has an upper cutoff approximately a thousand times smaller than the size of the GC (while their total mass is more than 10 times the mass of the GC). As $p$ approaches further to 


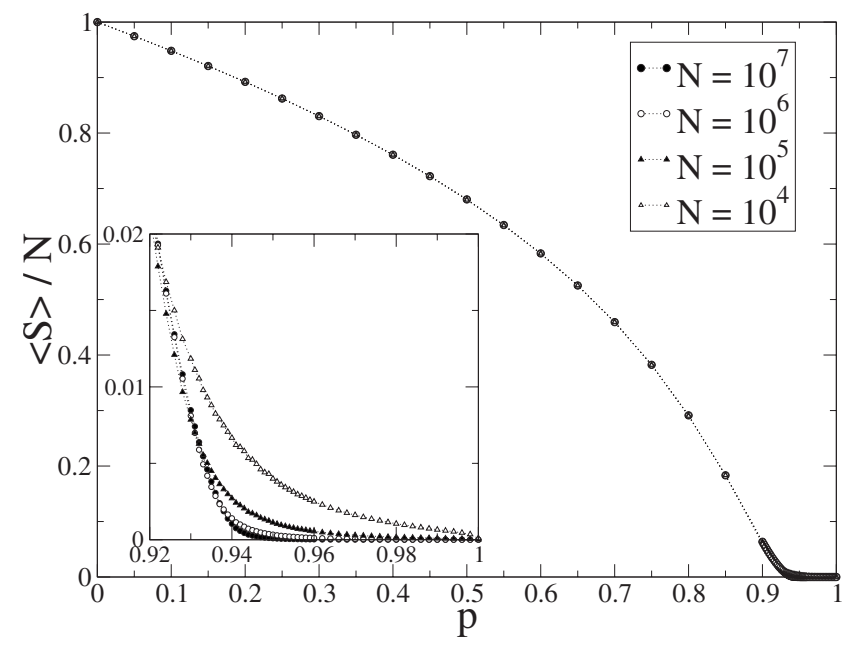

FIG. 2. The relative size $\frac{\langle S\rangle}{N}$ of the largest (giant) cluster in the network vs $p$. Different curves (which mostly overlap) correspond to a different value of $N$. The smaller window zooms into the region where the giant cluster appears.

unity, the relative size of the $\mathrm{GC}$ reaches zero at a critical value $p_{c} \simeq 0.946$ in a fashion which is subject to investigation here. We plot in Fig. 2 the relative size of the GC which demonstrates this behavior.

Determination of $p_{c}$. In order to proceed further, we first need to locate precisely the point where the GC emerges. As is clear from Fig. 2, this task is difficult to achieve on the basis of the presented data, since the average relative size $\langle S\rangle / N$ of the giant cluster approaches tangentially to the $p$ axis. Below, we propose a technique to circumvent this difficulty.

Consider not only the largest cluster but also the second largest one in the network, with respective sizes $S$ and $s$. The ratio $\langle s / S\rangle$ has the opposite trend as $\langle S\rangle / N$, i.e., it vanishes (as $N \rightarrow \infty$ ) for $p<p_{c}$ and is nonzero otherwise. We find that the transition is first-order-like in terms of this new parameter, i.e., the ratio $\langle s / S\rangle$ jumps from zero to a finite value at $p_{c}$. Figure 3 shows how the curves of $\langle s / S\rangle$ vs $p$ get steeper with increasing $N$.

We obtain the behavior in the limit $N \rightarrow \infty$ by extrapolating the data points corresponding to different values of $N$ along the (horizontal) lines of fixed $\langle s / S\rangle=\eta$. If the ratio jumps from zero to a finite value $\eta_{c}$ when $p=p_{c}$ (in the thermodynamic limit), then one expects that $\left[p_{c}-p_{\eta}(N)\right]$ $\rightarrow 0$ as $N \rightarrow \infty$, where $p_{\eta}(N)$ is the value of $p$ for a given $N$, at which $\langle s / S\rangle=\eta<\eta_{c}$. In fact, we show in Fig. 4 that the curves $p_{\eta}(N)$ for various values of $\eta$ are straight lines when plotted vs $N^{-1 / 4}$ with their linear extrapolations intersecting at a single point corresponding to $p_{c} \simeq 0.946$, precisely when $N^{-1 / 4}=0$. In addition to providing a precise numerical estimate of $p_{c}$, this method gives us a glance into the finite size effects in the system. Note in particular that the exponent $1 / 4$ sets a natural scale on the network size as a function of $p_{c}-p$ in the regime with $p<p_{c}$. This point is discussed later on in further detail.

We checked if the value we obtained for $p_{c}$ coincides with the well-known result for diluted networks [21]

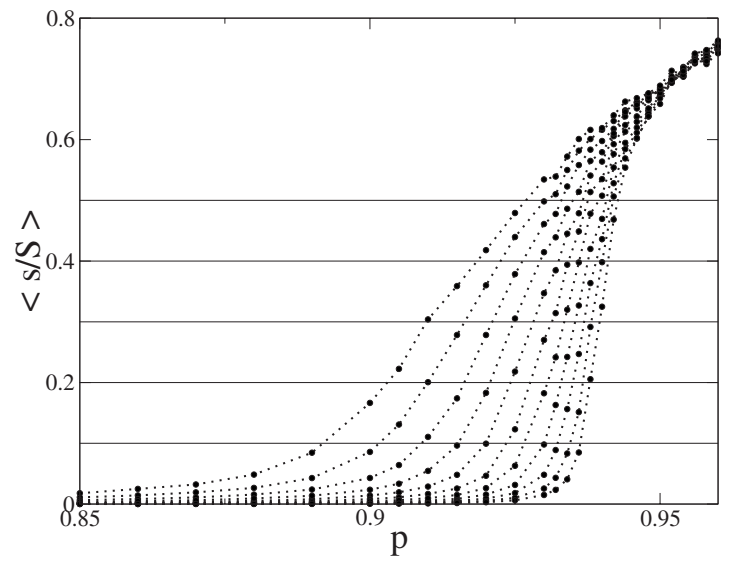

FIG. 3. The average value of $\frac{s}{S}$, the ratio of the size of the second largest cluster, $s$, to the size of the largest cluster $S$ in the network vs $p$ in the vicinity of the phase transition. Different curves correspond to different network sizes $N=2^{13}, 2^{14}, \ldots, 2^{23}$, with $N$ increasing from left to right in the figure.

$$
1-\bar{p}_{c}=1 /(\kappa-1), \quad \text { where } \kappa=\left\langle k^{2}\right\rangle /\langle k\rangle .
$$

Values corresponding to the network described here are $\kappa$ $=45.70$ and $\bar{p}_{c}=0.9776$, falling well outside the error margins for $p_{c}$ we obtained above (see Fig. 4). This agrees with our other observations that the transition analyzed here is distinct from the dilution-mediated percolation transition on fully grown networks.

\section{SIZE DISTRIBUTION OF FINITE CLUSTERS}

One can write down a master equation for the evolution of the cluster size distribution $N_{s}(t)$ as well. First we note that the clusters are treelike (numerically confirmed but not shown here), therefore the number of edges in a cluster of $s$ nodes is $s-1$. Then, the probability of connecting a new node to a cluster of size $s$ is $\pi_{s, t}=\frac{(2+A) s-2}{[2(1-p)+A] t}$, and the master equation for $m=2$ reads

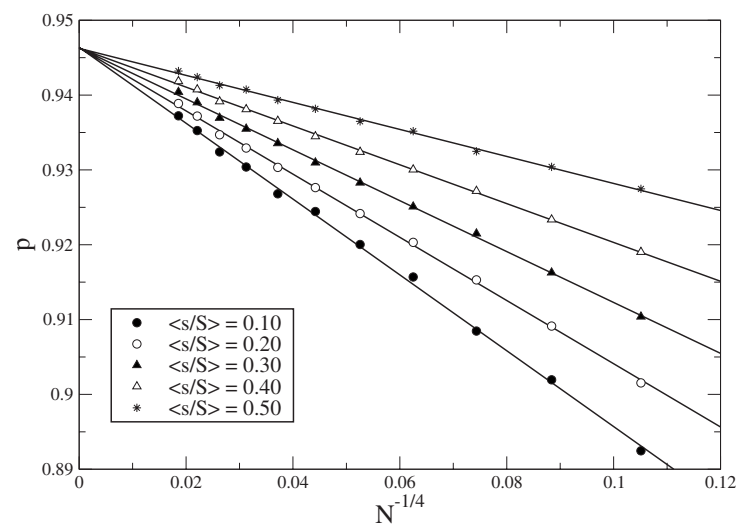

FIG. 4. Graphical determination of $p_{c}$. The linear extrapolation of the data points corresponding to different values of $\langle s / S\rangle$ (listed in the legend) meet in the thermodynamic limit $N \rightarrow \infty$ at $\langle s / S\rangle=0$ and $p_{c}=0.946$. 


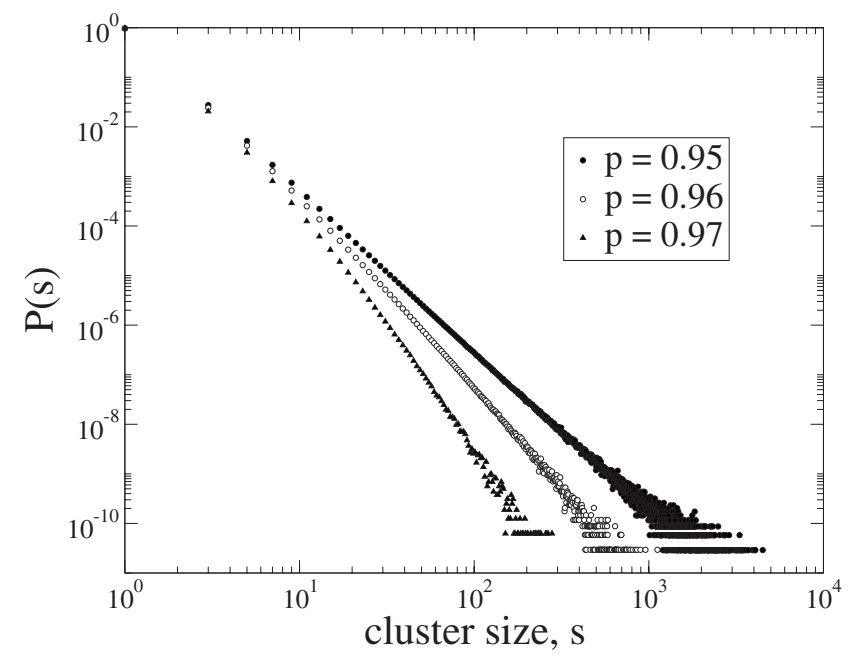

FIG. 5. The cluster size distribution for $p=0.95,0.96$, and 0.97 in the phase without the GC. Presented data is an average over many networks of size $N=2^{23}$.

$$
\begin{aligned}
N_{s}(t+1)= & N_{s}(t)+p \delta_{s, 1}-2(1-p) \pi_{s, t} N_{s}(t)+(1-p) \pi_{s, t}^{2} N_{s}(t) \\
& +(1-p)\left\{\sum_{j=1}^{s-2} \pi_{j, t} \pi_{s-j-1, t} N_{j}(t) N_{s-j-1}(t)\right. \\
& \left.+\pi_{s-1, t}^{2} N_{s-1}(t)\right\} .
\end{aligned}
$$

The first term on the right-hand side corresponds to the number of clusters of size $s$ at time $t$. The remaining terms reflect the change in this number at time $t$ due to the newly introduced node (successively) remaining isolated, connecting two clusters of size $s$, connecting twice to the same cluster of size $s$, joining two clusters of total size $(s-1)$, and connecting twice to the same cluster of size $(s-1)$. The analytical solution of this equation at steady state is not only cumbersome, but also fails to give insight into the finite size effects which is part of our focus here. We therefore resort to a numerical investigation of the model's percolative features.

We estimated the size distribution of finite clusters by simulating networks of size $N=2^{23}$ and averaging over 500 realizations. Let $P_{\mathrm{cl}}(s, N, p)$ be the probability for a randomly chosen cluster to be of size $s$. For $p=p_{c}$ and large $N$, we find $P_{\mathrm{cl}}(s, N, p) \sim s^{-3}$ as was obtained in Ref. [17] for a similar growing network. However, unlike the standard percolation phenomenon on diluted lattices, the finite cluster size distribution remains critical also away from $p_{c}$. This is demonstrated in Figs. 5 and 6 for $p>p_{c}$ and $p<p_{c}$, respectively. In Fig. 6 with $p<p_{c}$, the largest cluster is clearly isolated from the histogram obtained for the remaining smaller clusters. Note that the probability distribution of small clusters decays as a power law on both sides, however, the dependence on $p_{c}-p$, the distance from the percolation point, varies qualitatively on the two sides. Below, we quantify these aspects.

$$
\text { A. } p \geqslant p_{c}
$$

For $p \geqslant p_{c}$ (no giant cluster) the cluster size distributions for different values of $p$ maintain power-law tails, however,

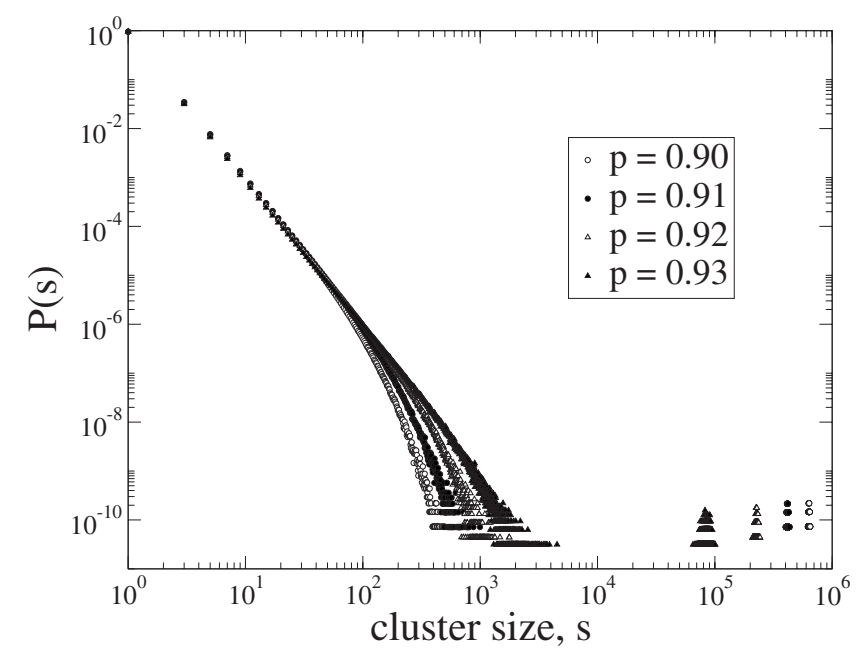

FIG. 6. The cluster size distribution for $p=0.90,0.91,0.92$, and 0.93 in the phase with the GC, obtained after averaging over many networks of size $N=2^{23}$. The disconnected portion of the distribution on the right is a single data point for each realization, representing the GC of the network.

with different slopes on a log-log scale. This indicates a $p$-dependent exponent, similar to the behavior of the degree distibution at low $p$ values. Based on this observation, we make a scaling ansatz for $P_{\mathrm{cl}}(s, N, p)$ for $p \geqslant p_{c}$ :

$$
P_{\mathrm{cl}}(s, N, p)=s^{-\tau(p)} f^{>}\left(\frac{s}{N^{D(p)}}\right),
$$

where $f>(x)$ is a scaling function, such that $f>(x \rightarrow 0)$ $=$ const and $f>(x \rightarrow \infty)=0$. In accordance with Fig. 5 we consider exponents $\tau$ and $D$ that depend on $p$. It should be noted that Eq. (6) is not the most general form of $P_{\mathrm{cl}}(s, N, p)$, and in particular, is inconsistent with the scaling of the GC in Ref. [17], where the approach to the critical point is found to be governed by a stretched exponential. In the present model, the ansatz in Eq. (6) was chosen based on the $q$ th-moment analysis [22] described below, a powerful technique that simultaneously confirms the validity of the functional form in Eq. (6) and provides numerical estimates for the exponents $\tau$ and $D$.

$q$ th-moment analysis. Given $P_{\mathrm{cl}}(s, N, p)$, consider the $q$ th moment of the cluster size $s$ :

$$
\left\langle s^{q}\right\rangle_{N}=\sum_{s=1}^{\infty} s^{q} P_{c l}(s, N, p),
$$

where we suppress the $p$ dependence of the exponents for brevity. Incorporating the ansatz in Eq. (6), and provided $N$ $\gg 1$ and $q>\tau-1$, one obtains, after some algebra, the dependence of $\left\langle s^{q}\right\rangle_{N}$ on $q$ as

$$
\begin{gathered}
N \gg 1 \\
\ln \left\langle s^{q}\right\rangle_{N} \stackrel{>}{=} \alpha(q) \ln N+\text { const, }
\end{gathered}
$$

where 


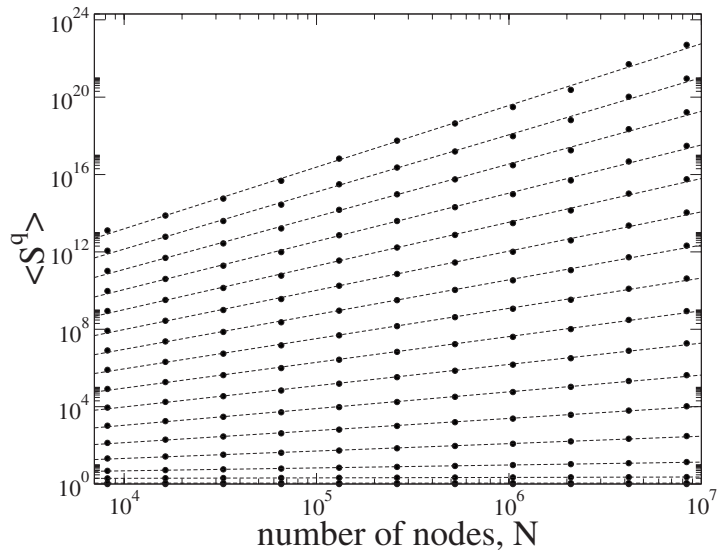

FIG. 7. $q$ th moments of the cluster size distribution for $p$ $=0.95$ and network sizes $N=2^{13}$ to $N=2^{23}$, with ascending powers of 2. Different curves correspond to $q=0,1,2,2.5,3,3.5, \ldots, 9$ with $q$ increasing vertically. Straight lines are linear fits to the data points, whose slopes have been used to obtain the graph in Fig. 8.

$$
\alpha(q)=(q-\tau+1) D .
$$

Therefore, the linearity of the curves $\ln \left\langle s^{q}\right\rangle_{N}$ vs $\ln N$, as well as the asymptotic linearity of the plot $\alpha(q)$ vs $q$ attest to the validity of the ansatz in Eq. (6).

Figure 7 shows the $q$ th moments as a function of the network size $N$ for $p=0.95$ on a $\log$-log scale. The slope of each data set gives $\alpha(q)$ which we plot next in Fig. 8. While the linearity of $\alpha(q)$ further confirms the validity of the ansatz in Eq. (6), the slope and the $x$ intercept of the linear asymptotes correspond, by virtue of Eq. (9), to the values $D$ and $\tau-1$, respectively. We observe that $\alpha(q)$ tends to a straight line with a slope of about $D=0.46$ and $x$ intercept around 2.1, corresponding to $\tau \simeq 3.1$. The asymptotes obtained for $\alpha(q)$ for $p=0.96$ and $p=0.97$ are also shown on the same figure in order to emphasize that the corresponding exponents $D$ and $\tau$ are indeed $p$ dependent.

$$
\text { B. } p<p_{c}
$$

When $p<p_{c}$, the balance between starting a new disconnected cluster versus joining existing clusters by extending

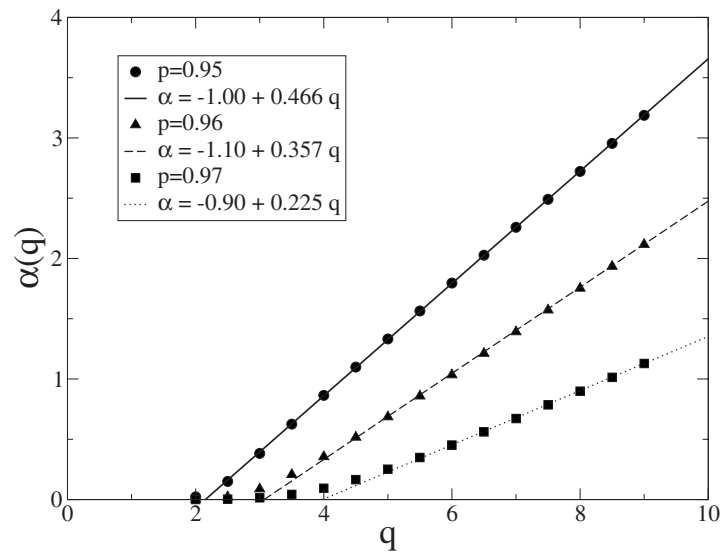

FIG. 8. $\alpha(q)$ defined in Eq. (8) and calculated from the slopes in Fig. 7 vs $q$, for $p=0.95,0.96,0.97$. For $q$ large enough, the data points fall on a straight line, confirming Eq. (9). out $m$ legs from the new node is tipped in favor of merging clusters. As a result, a single giant cluster with a macroscopic size emerges, while the rest of the network remains as a collection of finite clusters. Comparing Figs. 5 and 6, we notice that, unlike the case for $p>p_{c}$, deviations from $p_{c}$ do not alter the exponent $\tau=3$ of $P_{\mathrm{cl}}(s, N, p)$ in this region. Instead, they introduce, on the size distribution, a cutoff which gets sharper with increasing $p_{c}-p$. Hence, in presence of GC one observes a crossover due to a characteristic size $N_{x}$ set by the field $p_{c}-p$.

As is typical of crossover phenomena in the vicinity of a critical point, we observed upon inspecting networks of different sizes, that the cutoff on the size distributions is determined by either $N$ (when $N<N_{x}$ ) or $p$ (when $N>N_{x}$ ). While $N<N_{x}$, the system-yet unaware of the deviation from the percolation point-is still described by Eq. (6). After crossing over to $N>N_{x}$, the network looks no more critical. In fact, it is only then that the GC appears as distinctly larger than the rest of the clusters. Accordingly, we postulate two scaling regimes for $p<p_{c}$ :

$$
N \ll N_{x}(p): P_{\mathrm{cl}}(s, N, p)=s^{-\tau} f_{1}^{<}\left(\frac{s}{N^{D(p)}}\right) .
$$

This ansatz is similar to the scaling law in Eq. (6), except that $\tau$ no more depends on $p$ as suggested by our numerical analysis.

$$
N \gg N_{x}(p): P_{\mathrm{cl}}(s, N, p)=P_{\mathrm{cl}}(s, N \rightarrow \infty, p)=s^{-\tau} f_{2}^{<}\left(\frac{s}{\Delta p^{-1 / \sigma}}\right)
$$

following the finite size scaling arguments in standard percolation theory that were also adopted recently to study percolation on random networks [14].

Using the $q$ th-moment analysis this time on Eq. (11), we obtain for the regime $N \gg N_{x}(p)$

$$
\begin{gathered}
\Delta p \ll 1 \\
\left\langle s^{q}\right\rangle_{p}^{q>\tau-1} \stackrel{\sim}{\sim} \Delta p^{\beta(q)},
\end{gathered}
$$

where

$$
\beta(q)=-\frac{q-(\tau-1)}{\sigma},
$$

for $\Delta p \ll 1$ and $p>\tau-1$, while Eq. (8) is still valid in the region $N<N_{x}$. Note that $N_{x}$ for a given $p$ can be determined by simply plotting $\left\langle s^{q}\right\rangle_{p}$ vs $N$ for a sufficiently large $q$. One expects, based on Eq. (8) and Eq. (12), a power-law increase with $N$ up to $N \sim N_{x}$ and a constant function for $N>N_{x}$. Figure 9 shows the numerical evidence to this behavior for values of $p$ ranging from 0.86 to 0.94 for networks with sizes varying across three decades.

We estimated the value of $N_{x}$ for each $p$ by interpolating these curves and plotting against $p_{c}-p$, also shown in Fig. 9. The calculation of each data point in Fig. 9 requires intensive computational work, prohibiting a more detailed analysis. However, the estimate $\zeta=3.7 \pm 0.5$ for the crossover size exponent $N_{x} \sim \Delta p^{-\zeta}$ given in the inset of Fig. 9 is consistent with the value $-1 / 4$ employed in Fig. 4 for determining $p_{c}$. 


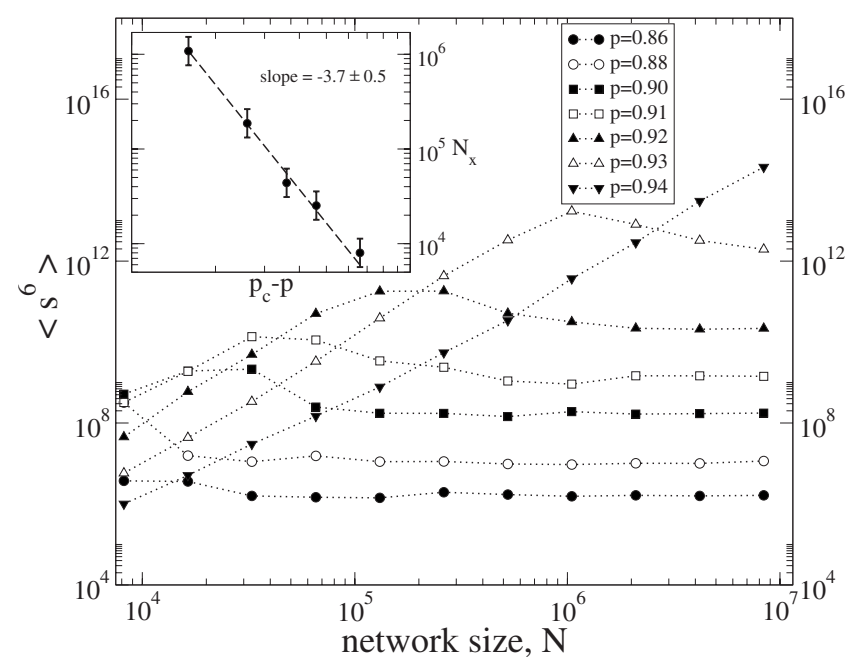

FIG. 9. 6th moment of the cluster size distribution vs the network size $N=2^{13}, 2^{14}, \ldots, 2^{23}$. Different values of $p$ are represented by different symbols. The peaks correspond to the crossover size $N_{x}$ for each $p$. The dependence of $N_{x}$ on the field $p_{c}-p$ is shown in the inset, where the error margin on the values of $\ln N_{x}$ corresponding to each peak is taken to be the sampling interval $\ln (2) \simeq 0.3$.

In other words, if we set the boundary between the critical and noncritical regimes as the borderline where $\langle s / S\rangle$ crosses over a preset value, then Fig. 4 shows that the network size $N_{x}$ at which this crossover takes place for some $p<p_{c}$ also scales as

$$
N_{x} \propto\left(p_{c}-p\right)^{-4}
$$

The above result also gives us an idea about the size requirements on a practical estimation of critical aspects in this model. In particular, we see that one does not need to get any closer to $p_{c}$ than that set by Eq. (13). Already for $p=0.94$, we fail to observe noncritical features even in the largest network we can practically simulate $\left(2^{23}\right.$ nodes $)$.

Finally, for values of $p$ satisfying $2^{23}>N_{x}(p)$, we check the scaling relation proposed in Eq. (11). Once again, we resort to the $q$ th moments of $\left\langle s^{q}\right\rangle$, this time as a function of $p$. Following the steps discussed earlier for Eq. (6), we obtain Fig. 10 which gives $\sigma \simeq 0.53$ and $\tau-1 \simeq 1.88$. Based on these values, we conjecture that for $p<p_{c}$ and $N>N_{x}$, the actual exponents are $\sigma=1 / 2$ and $\tau=3$. Below we suggest a scaling framework in which all our numerical findings form a consistent picture.

In fact, Eqs. (11) and (10) can be incorporated into a single scaling form

$$
P_{\mathrm{cl}}(s, N, p)=s^{-\tau} f^{<}\left(\frac{s}{\Delta p^{-1 / \sigma}}, \frac{s}{N^{D(p)}}\right),
$$

where $\Delta p=p_{c}-p$ and $f(x, y)$, for some constant $C$, behaves as

$$
f^{<}(x, y) \stackrel{x / y \gg C}{\sim} f_{1}^{<}(x)
$$

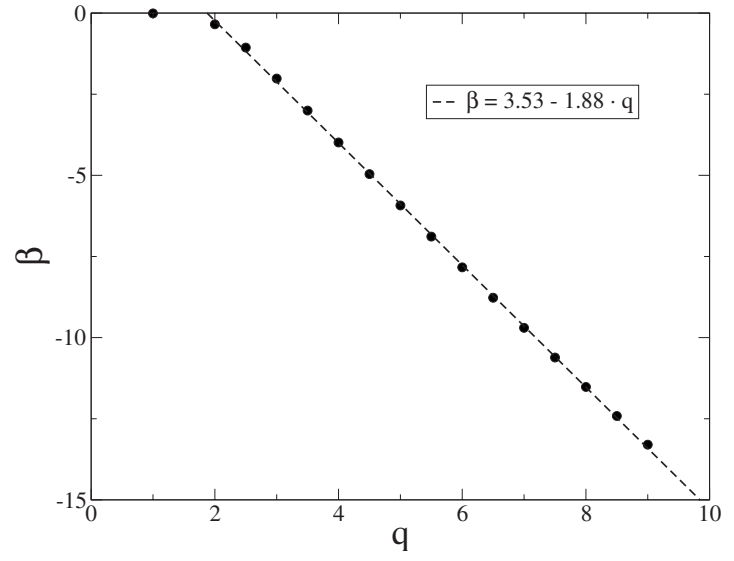

FIG. 10. $\beta(q)$ defined in Eq. (12) vs $q$. Data points are obtained from the $q$ th moments of the cluster size distribution for $p<p_{c}$ by using the procedure explained earlier for $p>p_{c}$. The exponents $\tau$ and $\sigma$ in Eq. (11) are obtained from this plot by using the relation $\beta(q)=-(q-\tau+1) / \sigma$.

$$
f^{<}(x, y) \stackrel{x / y \ll C}{\sim} f_{2}^{<}(y) .
$$

$f_{1}(x)$ and $f_{2}(y)$ are those given in Eqs. (11) and (10), respectively. The crossover between the two scaling limits is located at $\frac{x}{y} \sim C$ or $N_{x} \sim \Delta p^{-1 / \sigma D\left(p_{c}\right)}$. Combined with Eq. (13), this relates the three exponents obtained earlier by independent measurements

$$
1 / \zeta=\sigma D\left(p_{c}\right)
$$

and confirms the consistency of the two exponents $\sigma \simeq 1 / 2$ and $D\left(p_{c}\right) \simeq 1 / 2$ suggested on the basis of simulations, with the crossover size exponent $\zeta \simeq 4$ in Eq. (13) obtained by means of two independent measurements (Figs. 4 and 9).

Scaling of the giant cluster. Let us note that Eq. (11) has the same scaling form as in standard percolation on lattices. However, it is also well known that in this case the percolation probability $\pi$ (the probability that a randomly chosen node belongs to the infinite cluster) is given by another power law in the vicinity of the transition [23]:

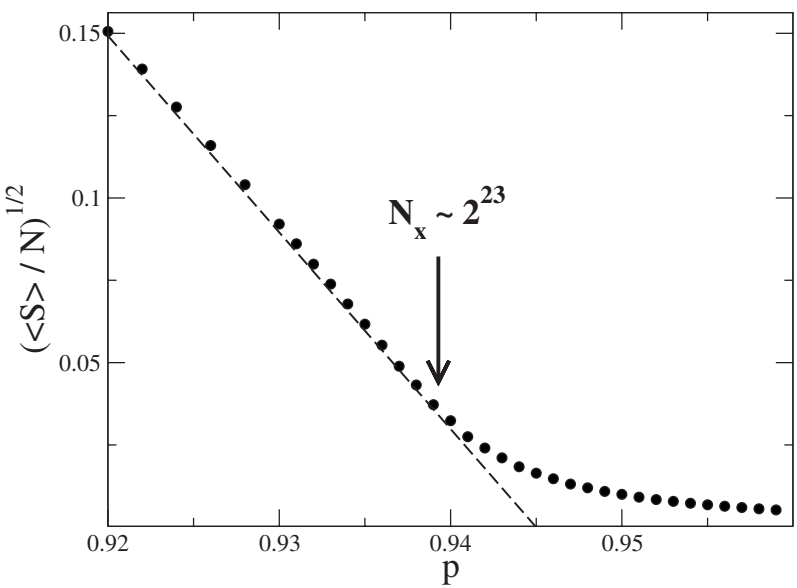

FIG. 11. Scaling of the GC given by Eq. (17) tested for $N$ $=2^{23}$. The linear decline towards $p_{c}$ survives up to the crossover point after which Eq. (11) is no longer valid. 


$$
\pi \sim \Delta p^{\beta} \text { with } \beta=\frac{\tau-2}{\sigma} .
$$

In our network, this probability is nothing but the quantity $\langle S\rangle / N$ given in Fig. 2. Therefore, we must have

$$
\langle S\rangle / N \sim(\Delta p)^{2}
$$

up to the values of $\Delta p$ for which $N>N_{x}$. In fact, plotting $\sqrt{\langle S\rangle / N}$ vs $p$, we find a convincing linear ascent toward zero, as long as $N \sim N_{x}(p)$, as demonstrated in Fig. 11 for $N=2^{23}$.

\section{CONCLUSION}

We presented a detailed numerical study of an imperfect network growth process which generates disconnected clusters that may grow by the addition of individual nodes or through mergers. We showed that the steady-state behavior exhibits a percolationlike transition, distinct from those observed in similar, earlier studied models. This points out that the critical features of cluster formation in such networks depends on the details of the dynamics. We also developed an original numerical method that allows one to determine the transition point with an accuracy sufficient to address near-critical phenomena, especially the finite size crossover behavior. All numerical data are consistent with the scaling ansatz summarized in Eqs. (6) and (14) and confirm the crossover network size exponent $\zeta \simeq 4$ in Eq. (13) governing the extent of the critical regime off $p_{c}$.

\section{ACKNOWLEDGMENT}

A.K. is grateful for the support from INFN.
[1] R. Albert and A.-L. Barabási, Rev. Mod. Phys. 74, 47 (2002).

[2] S. N. Dorogovtsev and J. F. F. Mendes, Evolution of Networks (Oxford University Press, Oxford, 2003).

[3] R. Pastor-Satorras and A. Vespignani, Phys. Rev. Lett. 86, 3200 (2001).

[4] C. Moore and M. E. J. Newman, Phys. Rev. E 61, 5678 (2000).

[5] A.-C. Gavin et al., Nature (London) 415, 141 (2002).

[6] J. Föster et al., Genome Res. 13, 244 (2003).

[7] N. Barkai and S. Leibler, Nature (London) 387, 913 (1997).

[8] W. Aiello, F. Chung, and L. Lu (unpublished).

[9] A. Kabakçıŏlu and A. L. Stella, Phys. Rev. E 72, 055102(R) (2005).

[10] M. P. H. Stumpf et al., Proc. Natl. Acad. Sci. U.S.A. 102, 4221 (2005).

[11] K. Hui and A. N. Berker, Phys. Rev. Lett. 62, 2507 (1989).

[12] R. Albert, H. Jeong, and A.-L. Barabási, Nature (London) 406, 378 (2000).

[13] P. Crucitti, V. Latora, M. Marchiori, and A. Rapisarda, Physica
A 320, 622 (2003).

[14] D. S. Callaway, M. E. J. Newman, S. H. Strogatz, and D. J. Watts, Phys. Rev. Lett. 85, 5468 (2000).

[15] R. Cohen, D. ben-Avraham, and S. Havlin, Phys. Rev. E 66, 036113 (2002).

[16] C. Moore and M. E. J. Newman, Phys. Rev. E 62, 7059 (2000).

[17] S. N. Dorogovtsev, J. F. F. Mendes, and A. N. Samukhin, Phys. Rev. Lett. 85, 4633 (2000).

[18] M. Boguña, R. Pastor-Satorras, and A. Vespignani, Eur. Phys. J.: Appl. Phys. 38, 205 (2004).

[19] B. Waclaw and M. Sokolov, Phys. Rev. E 75, 056114 (2007).

[20] A.-L. Barabási and R. Albert, Science 286, 509 (1999).

[21] R. Cohen, K. Erez, D. ben-Avraham, and S. Havlin, Phys. Rev. Lett. 85, 4626 (2000).

[22] C. Tebaldi, M. DeMenech, and A. L. Stella, Phys. Rev. Lett. 83, 3952 (1999).

[23] D. Stauffer and A. Aharony, Introduction to Percolation Theory (CRC, Boca Raton, 1994). 